\title{
Impulsive Noise Mitigation in Digital Subscriber Lines: the State-of-the-Art and Research Opportunities
}

\author{
Tong Bai, Student Member, IEEE, Hongming Zhang, Member, IEEE, Jiankang Zhang, Senior Member, IEEE, \\ Chao Xu, Member, IEEE, Anas F. Al Rawi, Member, IEEE, and Lajos Hanzo, Fellow, IEEE
}

\begin{abstract}
Digital subscriber lines (DSL) relying on twisted pairs and on home-passed fiber network, are still widely deployed in a large part of the world. However, its performance is severely restricted by the occurrence of impulsive noise. In this article, we present both the state-of-the-art and open research opportunities for impulsive noise mitigation in DSL. Its necessity is firstly discussed by briefly characterizing the impulsive noise and outlining the deleterious effects imposed on the system. Then, the state-ofthe-art is discussed by categorizing of the mitigation techniques into those at the transmitter as well as into parametric and nonparametric mitigation solutions at the receiver, followed by a detailed comparison in terms of mitigation efficiency, spectral efficiency, computational complexity and processing delay. Open research opportunities are discussed from the perspective of noise modeling for parametric mitigation, advanced mitigation design and machine-learning aided mitigation.
\end{abstract}

\section{INTRODUCTION}

The future home is envisaged to be a smart theater for the orchestration compelling of various personal multimedia and of machine-to-machine communications, for supporting diverse applications of entertainment, home automation and health care as well as home security and management. More explicitly, the devices accessing the home network will include not only communication devices (e.g. laptops, tablets and cellphones) and consumer electronics (e.g. televisions as well as radio and play stations), but also home appliances (e.g. washing machines and LED lamps). This real-time information aggregation paradigm imposes stringent requirements on the indoor communication systems exemplified by $\mathrm{Wi}-\mathrm{Fi}$, in terms of their throughput, reliability, delay and energy consumption.

As the backbone of indoor communications, the wired broadband access networks imposes an upper bound on wireless part of the home area networks in terms of their performance characteristics. Given their limits, the existing access networks tend to stifle the roll-out of the smart home services. Furthermore, the fiber-to-the-home (FTTH) concept, which is expected to satisfy the escalating demands in terms

L. Hanzo would like to acknowledge the financial support of the Engineering and Physical Sciences Research Council projects EP/Noo4558/1, EP/PO34284/1, COALESCE, of the Royal Society's Global Challenges Research Fund Grant as well as of the European Research Council's Advanced Fellow Grant QuantCom.

T. Bai, J. Zhang, C. Xu and L. Hanzo are with the School of Eletronics and Computer science, University of Southampton, SO17 1BJ, UK. H. Zhang is with Columbia University, New York, USA. Anas F. Al Rawi is with Research and Technology, British Telecom (BT), Adastral Park, Martlesham Heath, IP5 3RE, UK.

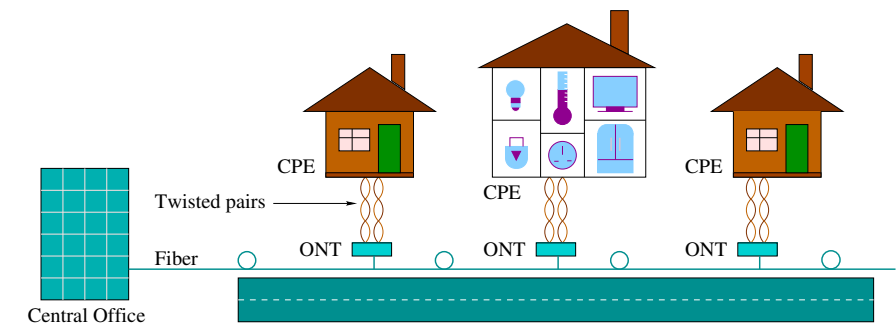

Fig. 1: Illustration of the network access in G.mgfast DSL [1] for supporting the forth coming smart home, where the concept of fiber-to-the-frontage (FTTF) is fulfilled both by home-passed fiber network and by the last drop of twisted pairs. The abbreviations are as follows: optical network terminal (ONT) and customer premise equipment (CPE).

of throughput and reliability as well as latency, is still not a ubiquitous reality at the time of writing and it is outright banned in protected historical architectural regions. To address this issue, G.mgfast [1] as shown in Fig. 1, the new digital subscriber line (DSL) technology, is associated with fiber-tothe-frontage (FTTF) architecture, which has been conceived for supporting high-quality broadband network access, relying on already-installed twisted pairs and the so-called homepassed fiber network.

Particular to the physical-layer implementation, the design of both modulation and coding schemes requires the detailed characterization of the noise properties. In contrast to wireless communication scenarios, the noise in DSL cannot be simply characterized by an additive white Gaussian noise (AWGN) model. More explicitly, the noise components in DSL can be generally classfied into crosstalk as well as impulsive and background noise. Crosstalk is the phenomenon by which a signal transmitted over a specific twisted-pair contaminates the signal in another twisted-pair. The multiuser crosstalk can be entirely averted by the fiber-to-thefrontage (FTTF) architecture [1] or efficiently mitigated by the transmit preprocessing techniques of vectoring and dynamic spectrum management [2]. However, the effects of impulsive noise continue to impose a substantial deleterious impact on DSL systems. For example, the amplitude of impulsive noise typically obeys a strongly non-Gaussian distribution, which is more concentrated around its mean value and has a heavier tail than the Gaussian distribution. Hence the classic demodulators originally optimized for the Gaussian distribution are unsuitable for these scenarios. Therefore, the detailed investigation of the impulsive noise in DSL constitutes an indispensable 
prerequisite for accommodating the aforementioned escalating demands.

Generally speaking, impulsive noise is a disturbance generated by a source that affects an electrical circuit by electromagnetic induction, electrostatic coupling or conduction. Specifically, impulses in DSL are typically caused both by switch-on/off of home appliances and by radio frequency interference imposed by aeronautical communications, satellite amateur communications, and FM radio broadcasting, etc. [3][5]. These impulses of noise can be generally classified into repetitive electrical impulse noise (REIN), single high impulse noise events (SHINE) and prolonged electrical impulse noise (PEIN) [5]. More explicitly, REIN appears periodically associated with a specific spectral envelope, while SHINE typically occurs in a transient pattern and PEIN commonly lasts for a certain period of time. In the literature, Henkel and Keßler [3] proposed an impulsive noise model based on their measurement campaign conducted for the ADSL standard associated with an operating frequency bands of upto $4 \mathrm{MHz}$ in 1994. Following this, Mann et al. [4] conceived a noise model for the VDSL standard, by extending the frequency band upto $30 \mathrm{MHz}$. In their recent work, Drooghaag et al. [5] explored the frequency range spanning upto $212 \mathrm{MHz}$ for characterizing the noise environment in the G.fast standard. In order to alleviate the influence of impulsive noise, various mitigation techniques have been proposed.

In this article, we commence by characterizing the influence of impulsive noise. Then we present the state-of-the-art in impulsive noise mitigation in DSL, both from the perspective of mitigation at the transmitter as well as from parametric and non-parametric mitigation at the receiver, followed by a comparison of the mitigation techniques, including their computational complexity, processing delay and spectral efficiency. Finally, promising research opportunities are discussed, focusing on noise modeling for parametric mitigation, advanced mitigation design and machine-learning aided mitigation.

\section{DSL TRANSCEIVER AND CHARACTERIZATION OF ITS IMPULSIVE NOISE}

In this section, we introduce the DSL transceiver and outline the detrimental effects of impulsive noise inflicted upon these transmission systems.

Fig. 2 illustrates the DSL transceiver, its low-pass channel and the noise process. At the transmitter, the information bits are typically protected by classic forward error correction (FEC) coding schemes. Upon passing through the interleaver, the FEC-encoded bits are then mapped to symbols by the symbol mapper. After the inverse discrete Fourier transform (IDFT)-based discrete multitone (DMT) modulation, the signals are transformed to the time domain (TD) and then transmitted over the channel, subject to channel impairments. At the receiver, the signals are firstly processed by the noise preprocessing unit and then transformed to the frequency domain (FD) after DFT-based DMT demodulation. Soft information is extracted from the symbol demapper and fed to the FEC decoder after deinterleaving. In order to ensue having a reliable link, once a packet is incorrectly received, a feedback flag is sent to the transmitter for requesting a retransmission. Finally, the information bits are FEC decoded.

The characterization of the impulsive noise in DSL is a prerequisite for designing efficient mitigation mechanisms. However, the diverse nature of sources and complex triggering mechanisms prohibit the derivation of analytic expressions. Hence, almost all existing empirical models rely on curve fitting based on measurement results. In general, impulsive noise can be modeled using the parameters of amplitude, impulse duration, impulse inter-arrival time (IAT) and spectral envelope, which are detailed as follows.

- Firstly, the empirical amplitude typically obeys a strongly non-Gaussian distribution [3]-[5]. More explicitly, compared to that of the Gaussian distribution having the same mean and variance values, the probability density function (PDF) of impulsive noise is more concentrated around its mean value and exhibits a longer tail. Hence the classic demodulators that were originally optimized for Gaussian noise environments, are unsuitable for these scenarios. The resultant unreliable soft information processed by the FEC decoder leads to a sub-optimal decoding, which often triggers a retransmission and prolongs the delay jitter.

- Secondly, the impulse duration ranges from microsecond to milliseconds. Specifically, the dominant impulses are typically shorter than $0.4 \mathrm{~ms}$ while a significant fraction of impulses are found to span upto $1.4 \mathrm{~ms}$ [5]. Bearing in mind that the duration of a DMT symbol is $20.83 \mu \mathrm{s}$ in the G.fast standard [2], a single impulse is long enough to contaminate several consecutive DMT symbols, potentially leading to dropping an entire packet. Moreover, the normalized time interval inflicting impulsive noise is about $3.29 \%$ of the total time-duration in the frequency band of VDSL standard [4]. Further considering that DSL transceivers have to operate in the face of an increasing number of noise sources in the recent standards of G.fast and of G.mgfast, impulsive noise is of relatively high occurrence probability, resulting in an unreliable DSL transmission link if no specific mitigation is invoked. Hence the system may fail to satisfy the backbone requirements of supporting high-integrity smart home networks.

- Thirdly, in their recent campaign, Drooghaag et al. [5] have shown that the impulsive noise is capable of achieving a maximum of $60 \mathrm{~dB}$ level over the background noise, albeit a gracefully decaying trend of its power spectral density is observed upon increasing the frequency. This may overwhelm the low-power transmitted signals. To address this issue, we have to transmit the signal using a much lower-order mapping scheme, which however degrades the throughput of DSL systems.

\section{Impulsive Noise Mitigation TeChniques}

In order to enhance the robustness of transmission systems against non-stationary impairments, various mitigation techniques have been proposed for DSL systems [6]-[12], for all the transceiver blocks seen in Fig. 2. As for the mitigation 


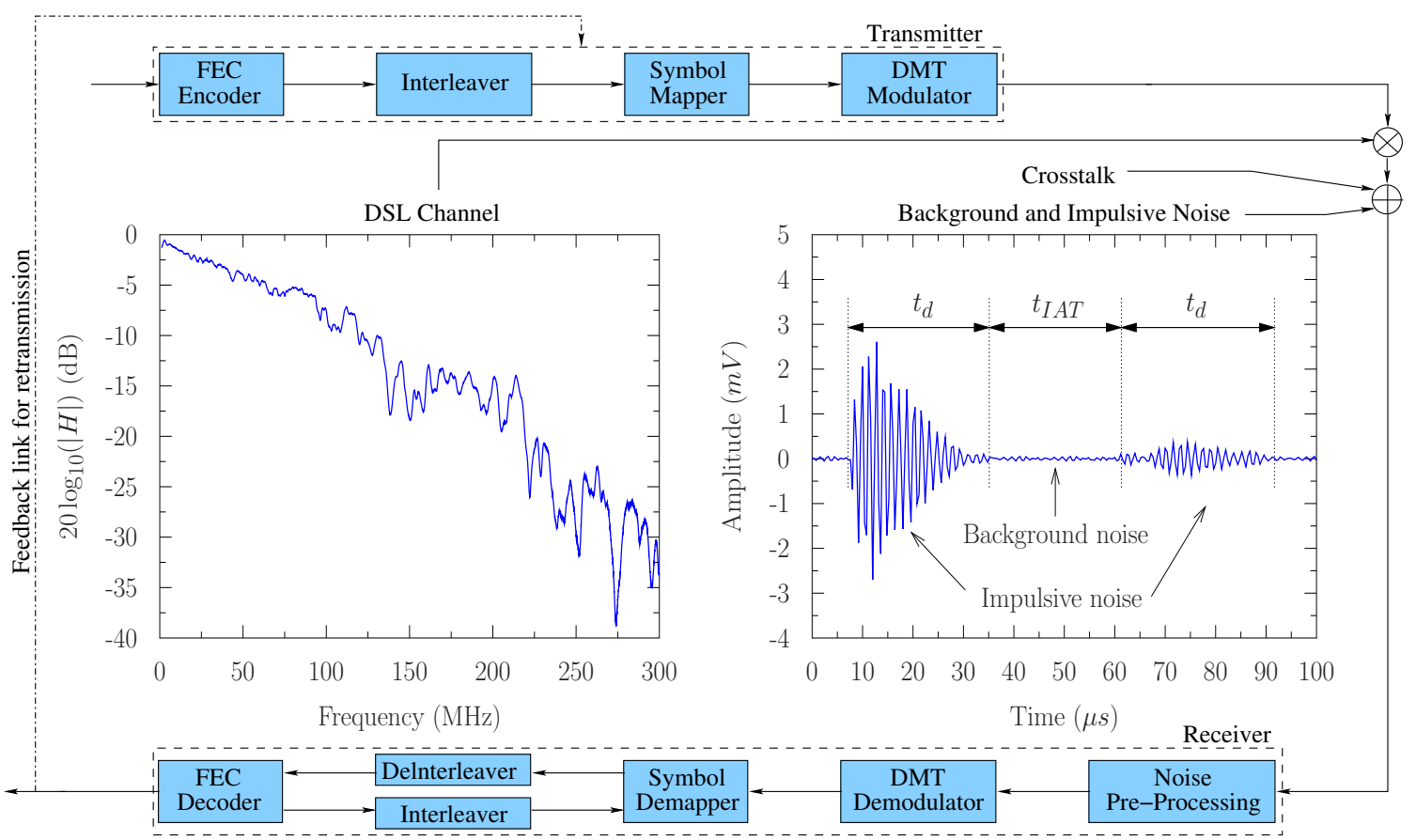

Fig. 2: The schematic of transceiver and the noise procedure in DSL, where $t_{d}$ refers to the time interval of impulse duration, whereas $t_{\mathrm{IAT}}$ represents impulse inter-arrival time (IAT). The associated abbreviations are as follows: FEC (Forward Error Correction) and DMT (Discrete Multi-Tone).

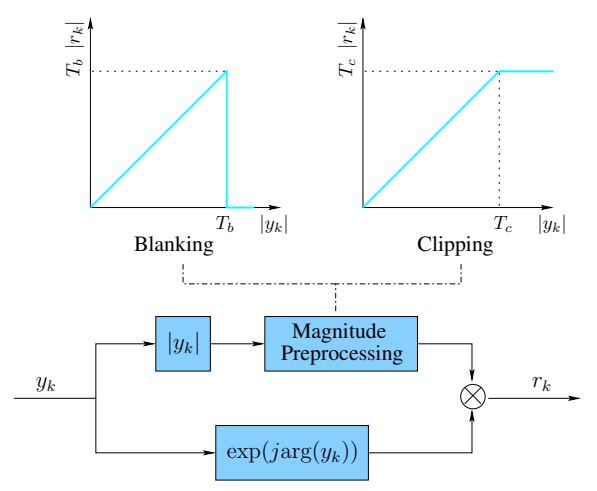

(a)

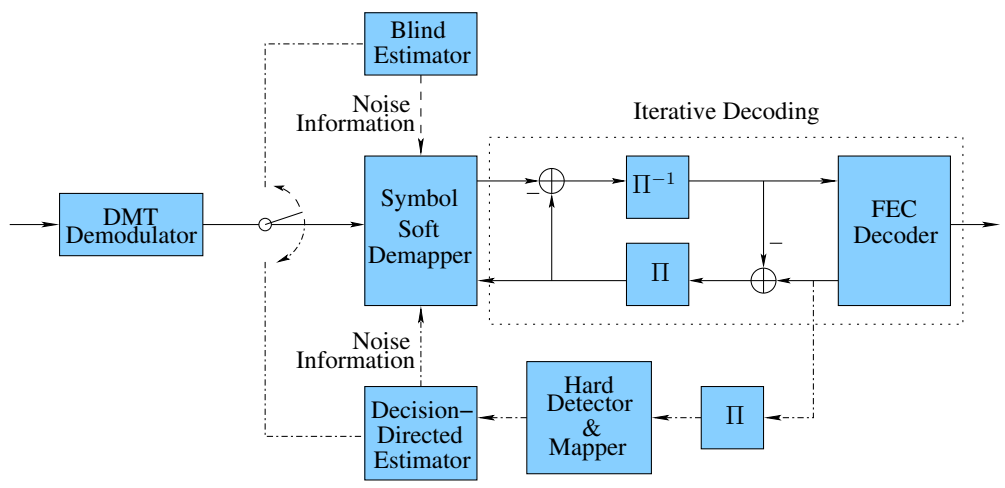

(b)

Fig. 3: Illustration of parametric impulsive noise mitigation at the receiver. a) Nonlinear pre-processing, where $T_{b}$ and $T_{c}$ represent the threshold amplitude of the blanking and clipping schemes, respectively, while $\left|y_{k}\right|$ and $r_{k}$ refer to the signal before and after pre-processing, respectively. b) The iterative impulsive noise estimation and data detection scheme. The associated abbreviations are as follows: FEC (Forward Error Correction) and DMT (Discrete Multi-Tone).

techniques used at the receiver, we further classify them into parametric and non-parametric approaches, according to whether the statistical knowledge of the noise is required.

\section{A. Approaches at the Transmitter}

The impulsive noise mitigation at the transmitter side may rely on channel coding and interleaving as well as on automatic-repeat-request (ARQ), as detailed below.

1) Channel Coding: The errors caused by high-power impulses have to be corrected. To elaborate, a concatenation of long Reed-Solomon (RS) codes and trellis coded modulation (TCM) has been recommended in the recent G.fast standard [2]. Since both components of this standardized scheme are out-of-date at the time of writing, capacity-approaching coding schemes such as turbo codes and low density parity check
(LDPC) codes are also considered for the next generation of DSL. As a benefit of their iterative decoding structure, these capacity-approaching coding techniques are capable of achieving substantial coding gains over the above-mentioned concatenated schemes.

2) Interleaving: Given that it typically lasts for a prolonged period of time, impulsive noise commonly inflicts numerous consecutive errors, which are likely to exceed the correction capability of FEC codes. Bit- or symbol-interleaving seen in Fig. 2 can be invoked for randomizing the errors. As a result, the randomized errors may be more readily corrected. Apart from improving the error correction performance of FEC codes, interleaving is also capable of directly alleviating the impulsive noise effects. To elaborate, if impulsive noise is inflicted in the TD, this will be spread and averaged over all samples in the FD after the DFT-based demodulation. 
As a result, increasing the number of contaminated samples in a DMT symbol directly increases the noise level of this DMT symbol. In other words, the number of contaminated TD samples in a DMT symbol determines the receive signal-tonoise ratios (SNRs) of different DMT symbols. Hence, an error floor is likely to occur, if receive SNR of some DMT symbols is below the threshold required for correct DMT demodulation. In order to uniformly disperse the number of contaminated samples in each DMT symbol, a TD interleaver was proposed [6] by placing the interleaver after the IDFT block of the DMT modulation. As a result, the impaired samples can be spread over a larger number of impulse-free samples and hence the error floor can be mitigated. However, the operation of interleaving and deinterleaving typically prolongs the delay, which may adversely affect some delay-sensitive applications, such as online gaming.

3) $A R Q:$ As a benefit of its simple mechanism, ARQ constitutes a popular error control method for channels contaminated by impulsive noise. To elaborate, once a packet is unsuccessfully received due to contamination by impulsive noise, a negative acknowledgement flag is fed back to the transmitter and then the original message is resent until it is correctly received, or a full-buffer status is reported, or alternatively the maximum tolerable round-trip delay time is reached.. In this way, having a reliable transmission link is guaranteed. However, the additional overhead and delay jitter caused by retransmission is increased but only when impulsive noise occurs. Particular to DSL systems, ARQ is demonstrated to be capable of significantly improving the effective throughput in cooperation with low-density paritycheck codes as well as by using the concatenation of ReedSolomon codes and trellis coded modulation [7].

\section{B. Parametric Approaches at the Receiver}

Given that the impulsive noise component of REIN appears periodically associated with a specific spectral envelope, we may estimate its parameters using some pilot overhead during the training stage and then optimize the parametric mitigation approaches at the receiver accordingly. The typical parametric mitigation approaches include adaptive filtering, nonlinear preprocessing as well as iterative symbol detection and decoding, as detailed below.

1) Adaptive Filtering: Colored nature has been observed in impulsive noise [5], which exhibits extremely high power at some frequencies and low power at others. In order to attain successful information recovery, a high transmit power is required for the subchannels contaminated by high-power noise, which is however infeasible due to the restriction of power masks. To address this issue, an adaptive filter [8] can be conceived for whitening the colored noise. Specifically, some cyclostationary noise components occur periodically associated with a deterministic PSD in DSL systems. By characterizing them using a linear periodic time-variant model, we may accordingly design an appropriate filter for whitening the noise. As a benefit, the signals can be correctly received using a transmit power below the power mask.
2) Nonlinear Pre-processing: Nonlinear pre-processing may be used for constraining the received signal within predefined magnitude boundaries while retaining the same phase [9]. Specifically, as shown in Fig. 3a, when the magnitude exceeds the threshold, we force it either to be zero or to the threshold value relying on so-called blanking and clipping schemes, respectively. More particularly, the threshold optimization in nonlinear pre-processing strikes a trade-off between reliable detection and low false alarm probability, because the useful signals have to be preserved, while the impulsive noise has to be mitigated. An effective approach is to find the maximum signal-to-noise ratio value at the output of the nonlinear preprocessing block under diverse threshold values, based on the statistical knowledge of the noise.

3) Symbol Demapper and Iterative Decoding: The nonGaussian nature of the impulsive noise degrades the optimality of the symbol detector that was originally designed for the classic AWGN channel. To tackle this issue, the Gaussian distribution based expression of the a posteriori probability in the maximum a posteriori (MAP) demapper has to be replaced by a new PDF formula. The updated log-likelihood ratios (LLRs) of the a posteriori probability can then be fed into an iterative FEC decoder for error correction. As a more advanced approach, the process of impulsive noise estimation and symbol detection as well as iterative decoding is conceived in an iterative manner [10]. To elaborate a little further with the aid of Fig. 3b, the initial estimated information of the impulsive noise gleaned from the blind estimator is invoked by both the symbol demapper and the iterative decoder, whose outputs are re-modulated and fed back to the decision-directed estimator for assisting the noise estimation. In this way, the overall system performance is significantly improved after a few iterations.

\section{Non-Parametric Approaches at the Receiver}

Generally, taking into account the statistical characteristics of noise requires considerable overheads, which leads to a degradation of the bandwidth efficiency. Hence, the nonparametric approaches, that are based on simultaneous observation, have attracted a growing interest. Typically, the existing non-parametric mitigation approaches include erasure decoding and compressed-sensing aided mitigation, as detailed below.

1) Erasure Decoding: In a scenario where the statistical characteristics of the noise are unknown, once a symbol is contaminated by impulsive noise, the corresponding LLRs gleaned from the soft demapper become unreliable. As a result, errors may occur in those contaminated symbols and then be propagated to their neighbouring symbols during iterative decoding. A promising solution is to identify these contaminated symbols and then to erase them before FEC decoding. More explicitly, once a symbol is infested by an impulse, its magnitude will be increased. In this case, we are able to single them out and then mark them as erasures [11]. As a benefit, the LLRs corresponding to impulsive-noise-impaired symbols are not invoked in the FEC decoding process as extrinsic information and hence the iteration-induced errors may be 


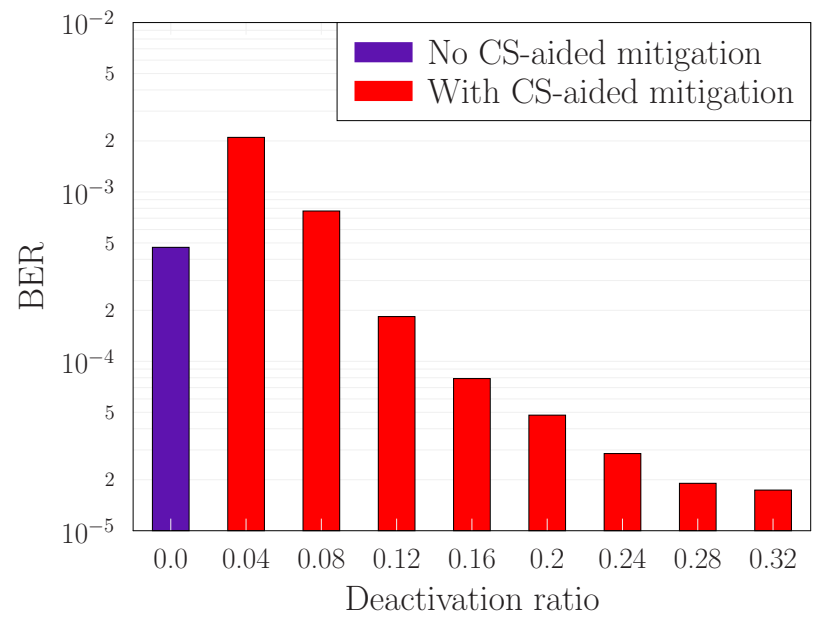

Fig. 4: Bit error ratio (BER) performance versus normalized pilot number of compressed sensing (CS)-aided impulsive noise mitigation, where the quadrature phase shift keying (QPSK) is used and $t_{d} / t_{\mathrm{IAT}}=0.001$. The power ratio of impulsive-to-background noise is $50 \mathrm{~dB}$ and the signal-tobackground-noise ratio is $35 \mathrm{~dB}$. DMT is the abbreviation of Discrete MultiTone.

avoided. The erased symbols may then be filled in during the later iterations. However, if the codeword is deemed to be corrupted on the basis of its mutual information between the soft- and hard-decision symbols, an ARQ request is activated for retransmission, before sending a new codeword.

2) Compressed-Sensing Aided Mitigation: In practical DSL systems, some high-attenuation frequency-domain subchannels are barred from data transmissions. However, we still receive impulsive noise during these nulled subchannels, hence we can reconstruct the impulsive noise using compressedsensing (CS) based on sparse reconstruction [12]. Specifically, we transform the consecutive impulses into a discontinuous form with the aid of TD interleaving [6], which can be considered as a sparse vector. After the DFT-based demodulation, the signals are projected to the specific space constituted by the subchannels that are deactivated, which is associated with a reduced dimensionality. Then the reconstruction problem can be formulated as an $\ell_{1}$-Norm or a mixed $\ell_{2} / \ell_{1}$-Norm minimization problem, which can be readily solved with the aid of estimating its support and its impulse amplitude. In this case, the impulses can be directly mitigated by subtracting the reconstructed samples. Let us define the deactivation ratio as the number of deactivated subchannels to the total number of subchannels. In Fig. 4, we compare the BER performance versus the deactivation ratio of the conventional detector and of the detector equipped with CS-aided mitigation. It can be readily observed that CS-aided mitigation is capable of improving the BER performance for a deactivation ratio in excess of about 0.1 . This deactivation process imposes certain spectral efficiency erosion.

\section{Comparison of Mitigation Techniques}

Table I compares these impulsive noise mitigation techniques from the perspective of the target noise type, mitigation performance, spectral efficiency, computational complexity and processing delay. It is plausible that the various DSL system characteristics of Table I tend to require a compromise. For example we can always improve the performance by invoking more complex signal processing techniques. However, this often imposes a higher delay jitter for example due to using more FEC-coding iterations and/or more ARQ retransmission attempts. Naturally, the above characteristics also depend on the type of impairments to be mitigated. Future system design should ideally aim for finding all the optimal operating points of DSL systems where none of the abovementioned characteristics can be improved without degrading at least one, but typically several of these features. The collection of these optimal operating points constitutes a socalled Pareto-front in multi-component optimization.

\section{Open Research Opportunities}

\section{A. Noise Modeling for Parametric Mitigation}

Having statistical knowledge concerning the impulsive noise is a prerequisite for parametric mitigation techniques. Therefore, the characteristics of impulsive noise have to be further investigated in the new environment, for supporting nextgeneration DSL in the smart home. Firstly, as shown in Fig. 1, the optical network terminal (ONT) will be deployed closer to the CPE at home, moving it from the conventional fiber-tothe-distribution-point (FTTdp) to the FTTF, which may modify the noise's amplitude, the duration and the IAT distributions at the ONT. Secondly, the forthcoming smart home network is expected to support diverse appliances and sensors, whose switch-on/off operations potentially increase the impulsive noise imposed on the CPE. Thirdly, in order to enhance the throughput, we could aim for extending the operational spectrum upto $500 \mathrm{MHz}$. However, the system would suffer from increased impulsive noise. Fourthly, the existing singleinput single-output (SISO) transmission is expected to be replaced by advanced multiple-input multiple-output (MIMO) techniques, which impose spatial correlation on the impulsive noise. To elaborate, the existing SISO transmission is realized by a differential mode (DM), relying on a single twisted pair. This actually constitutes a waste of resources, because in the final drop from the frontage to home, often two twisted pairs are available due to the historical installment of telephone lines. In fact, these two pairs can be bonded together, hence resulting in an additional physical link. Moreover, the advent of the so-called phantom mode (PM) [13] enables us to create a "virtual" data stream, by mapping data using the difference between the two common mode signals on both twisted pairs. Since both pairs are physically adjacent to each other, the impulsive noise is correlated spatially due to the coupling effect. In this case, the impulsive noise of MIMO transmission becomes correlated in the time-, frequency- and spatialdomain, which is worthy of further analysis. Furthermore, in contrast to the conventional DSL data transmission relying on the recent concept of "transmission-line mode" proposed by Cioffi et al. [14] exploited a so-called "waveguide mode" in DSL by using sub-millimeter-wave and millimeter-wave frequency bands for achieving a Terabit-level data rate, where both the excitation and the dominant factors of impulsive noise may be substantially reshaped. 
TABLE I: Comparison of impulsive noise processing techniques, where Tx/Rx refers to the transmitter or the receiver, whereas ARQ corresponds to automaticrepeat-request and CS means compressed sensing.

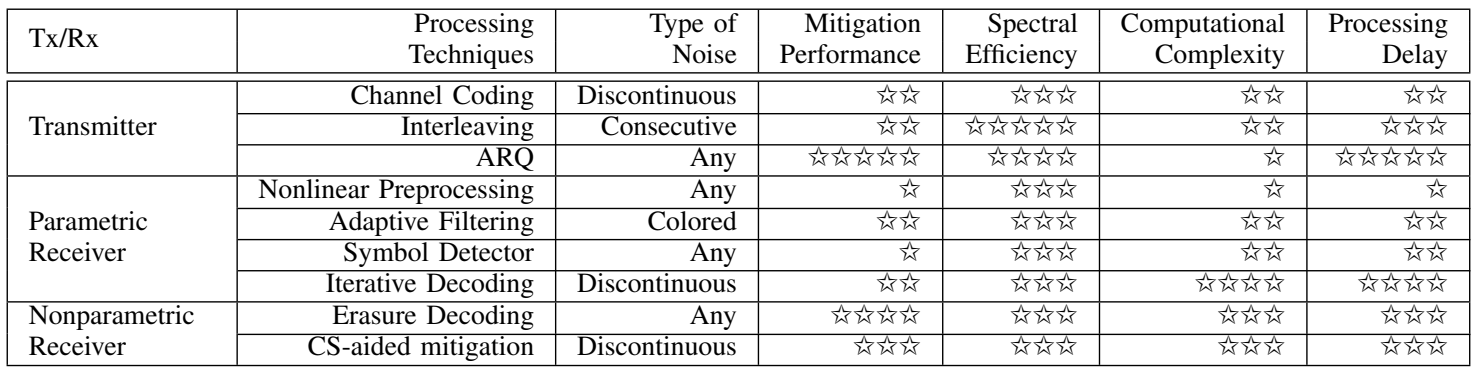

\section{B. Advanced Mitigation Design}

Given that impulsive noise emerging from diverse sources may exhibit different behaviors in terms of its amplitude, duration, IAT and spectrum in practice, there is no universally applicable mitigation technique that can eliminate all these noise components. To address this issue, hybrid mitigation schemes can be conceived. For example, CS-based impulsive noise detection can also be integrated into erasure based decoding, for the sake of identifying the specific impaired symbols. Moreover, unreliable soft information of the symbols impaired by high-magnitude impulses may also lead to unsuccessful decoding, which can be alleviated by incorporating nonlinear pre-processing that is capable of restricting the magnitude of received signals. Furthermore, the characteristics of impulsive noise are dominantly influenced by the sources of interference in the vicinity of the twisted-pair, which makes its properties specific to the operational site [5]. Therefore, both impulsive noise monitoring and the per-line configuration of impulsive noise parameters are capable of substantially enhancing the mitigation performance.

\section{Machine-Learning Aided Noise Mitigation}

The signal processing techniques in communication systems have solid statistical and information theoretical foundations, which are often accompanied by tractable mathematical models, which usually obey linear, stationary and Gaussian statistics. By contrast, the occurrence of impulsive noise in DSL introduces non-stationary and intractable factors. Hence, a machine-learning (ML) based communication system that does not require a tractable mathematical model may be capable of improving the attainable performance. On one hand, ML-based methods can be utilized for improving the noise modeling accuracy. For example, the temporal characteristics of SHINE in the home typically obey a hidden-Markov process characterized by the Poisson distribution. In this context, MLbased algorithms can be used for estimating its parameters. On the other hand, inspired by the concept of "autoencoder" [15], we may directly apply ML to the physical layer, by completely replacing the existing communication system. To elaborate, the chain of multiple independent blocks (channel codec, modem, etc.) can be replaced by a single deep-learning (DL)-based black box. Beneficially, we may improve the performance by exploiting the joint optimization capability of such a DL-based black box. Moreover, the statistical knowledge of both the channel and of the noise is no longer a prerequisite. Hence, the corresponding overheads can be avoided, resulting in an enhanced spectral efficiency.

\section{CONCLUSions}

In this article, the state-of-the-art in impulsive noise mitigation in DSL has been presented complemented by a range of open research opportunities. The necessity of impulsive noise mitigation was investigated by briefly portraying the detrimental effects of impulsive noise. The state-of-the-art has been discussed by categorizing of the mitigation techniques into those at the transmitter as well as into parametric and nonparametric mitigation solutions used at the receiver, followed by a detailed comparison. Open research opportunities have also been listed from the perspective of noise modeling as well as advanced mitigation and ML-aided paradigms. In a nutshell, the impulsive noise processing in DSL still constitutes an attractive research area for improving the performance of the DSL system in the face of impulsive noise.

\section{REFERENCES}

[1] W. Coomans, R. B. Moraes, K. Hooghe, A. Duque, J. Galaro, M. Timmers, A. J. van Wijngaarden, M. Guenach, and J. Maes, "XG-fast: the 5th generation broadband," IEEE Communications Magazine, vol. 53, pp. 83-88, Dec. 2015.

[2] P. Odling, T. Magesacher, S. Host, P. O. Borjesson, M. Berg, and E. Areizaga, "The fourth generation broadband concept," IEEE Communications Magazine, vol. 47, pp. 62-69, Jan. 2009.

[3] W. Henkel and T. Keßler, "A wideband impulsive noise survey in the German telephone network: statistical description and modeling," Archiv fur Elektronik und Ubertragungstechnik, vol. 48, no. 6, pp. 277-288, 1994.

[4] I. Mann, S. McLaughlin, W. Henkel, R. Kirkby, and T. Kessler, "Impulse generation with appropriate amplitude, length, inter-arrival, and spectral characteristics," IEEE Journal on Selected Areas in Communications, vol. 20, pp. 901-912, June 2002.

[5] B. Drooghaag, J. Maes, M. E. Fani, and V. Moeyaert, "Exploring field noise on G.fast frequencies," in Proceedings of IEEE GLOBECOM 2017, pp. 1-6, Dec. 2017.

[6] A. Al-Dweik, A. Hazmi, B. Sharif, and C. Tsimenidis, "Efficient interleaving technique for OFDM system over impulsive noise channels," in Proceedings of IEEE PIMRC 2010, pp. 167-171, Sep. 2010.

[7] J. Neckebroek, M. Moeneclaey, M. Guenach, M. Timmers, and J. Maes, "Comparison of error-control schemes for high-rate communication over short DSL loops affected by impulsive noise," in Proceedings of IEEE ICC 2013, pp. 4014-4019, June 2013.

[8] B. Lee, J. M. Cioffi, S. Jagannathan, and M. Mohseni, "Gigabit DSL," IEEE Transactions on communications, vol. 55, pp. 1689-1692, Sep. 2007.

[9] I. Al-Neami, G. A. Al-Rubaye, M. Johnston, and C. Tsimenidis, "Optimal threshold calculation for improved impulsive noise mitigation in the frequency domain," in Proceedings of IEEE 11th CSNDSP, pp. 1-5, July 2018. 
[10] T. Bai, C. Xu, R. Zhang, A. F. Al Rawi, and L. Hanzo, "Joint impulsive noise estimation and data detection conceived for LDPC-coded DMTbased DSL systems," IEEE Access, vol. 5, pp. 23133-23145, Oct.. 2017.

[11] Y. Zhao, K. Ho-Van, and T. Le-Ngoc, "Efficient erasure marking technique for interleaving delay reduction in DSL systems impaired by impulse noise," in Proceedings of IEEE ICC 2012, pp. 3170-3174, June 2012.

[12] A. A. Quadeer, M. S. Sohail, and T. Y. Al-Naffouri, "A compressed sensing based method with support refinement for impulse noise cancelation in DSL," in Proceedings of IEEE 14th SPAWC, pp. 255-259, June 2013.

[13] W. Foubert, C. Neus, L. V. Biesen, and Y. Rolain, "Exploiting the phantom-mode signal in DSL applications," IEEE Transactions on Instrumentation and Measurement, vol. 61, pp. 896-902, Apr. 2012.

[14] J. M. Cioffi, K. J. Kerpez, C. S. Hwang, and I. Kanellakopoulos, "Terabi DSLs," IEEE Communications Magazine, vol. 56, pp. 152-159, Nov. 2018.

[15] S. Drner, S. Cammerer, J. Hoydis, and S. t. Brink, "Deep learning based communication over the air," IEEE Journal of Selected Topics in Signal Processing, vol. 12, pp. 132-143, Feb. 2018.

Tong Bai (S'15) received the B.Sc. degree in telecommunications from Northwestern Polytechnical University, Xi'an, China, in 2013, the M.Sc. and Ph.D. degrees in wireline and wireless communications from University of Southampton, U.K. in 2014 and 2019, respectively. His research interests include performance analysis, transceiver design and utility optimization both for wireline and for wireless communications.

Hongming Zhang (S15-M17) received the B. Eng. degree (with first class honors) in telecommunications from the Nanjing University of Aeronautics and Astronautic (NUAA) and the City University London, both in 2011, the M. Sc. and Ph.D. degrees in wireless communications from University of Southampton in 2012 and 2017, respectively. His research interests include coding and modulation techniques, compressed sensing with applications to wireless communications, and heterogeneous networks.

Jiankang Zhang (S08-M12-SM'18) received the Ph.D. degree in Communication and Information Systems from Zhengzhou University in 2012. Dr. Zhang has been a lecturer from 2012 and an associate professor from 2013 in School of Information Engineering, Zhengzhou University. Since 2014, he has been a senior research fellow in the University of Southampton, UK. His research interests are in the areas of wireless communications and signal processing, aeronautical communications and broadband communications.

Chao Xu (S'09-M'14) received the B.Eng. degree from Beijing University of Posts and Telecommunications, China, and Queen Mary, University of London, UK, both in 2008. He obtained his MSc and Ph.D. degrees in Wireless Communications from the University of Southampton, UK in 2009 and 2015, respectively, where he is currently a postdoctoral researcher. His research interests include reduced-complexity MIMO design, noncoherent detection, extrinsic-information-transfer-chart-aided turbo detection, and cooperative communications.

Anas F. Al Rawi (S'08-M'13) received the Ph.D. degrees in communications and signal processing from Newcastle University, U.K., in 2011. From 2011 to 2012, he was a Postdoctoral Researcher with Swansea University and Queens University Belfast. He is currently a Research Manager in network physics with BT labs, and a Royal Society Industry Fellow with the Cavendish Laboratory, University of Cambridge. His research interests include computational electro-magnetics, transmission cross-layer optimization, and cooperative and MIMO systems.
Lajos Hanzo [F04] FREng, FIEEE, FIET, Fellow of EURASIP, DSc, received his degree in electronics in 1976 and his doctorate in 1983. He holds an honorary doctorate from the Technical University of Budapest (2009) and from the University of Edinburgh (2015). He is a member of the Hungarian Academy of Sciences and a former Editor-in-Chief of the IEEE Press. He is a Governor of both IEEE ComSoc and of VTS. 